\title{
Effect of Thermo-Mechanical Treatments on the Microstructure and Mechanical Properties of the Metastable $\beta$-type Ti-35Nb-7Zr-5Ta Alloy
}

\author{
Athos Henrique Plaine ${ }^{a} *$, Murillo Romero da Silva ${ }^{a}$, Claudemiro Bolfarini ${ }^{a}$ \\ ${ }^{a}$ Departamento de Engenharia de Materiais, Universidade Federal de São Carlos, Rod. Washington \\ Luis Km 235, 13565-905, São Carlos, SP, Brasil
}

Received: July 02, 2018; Revised: September 20, 2018; Accepted: September 28, 2018

\begin{abstract}
In this work, theoretical composition design and thermo-mechanical treatments were combined in order to improve the mechanical compatibility of a biomedical $\beta$-type titanium alloy. By applying a composition design theory, cold rolling and low temperature aging, a metastable $\beta$-type Ti-35Nb-7Zr$5 \mathrm{Ta}(\mathrm{wt} \%)$ alloy with an elastic modulus of $47 \mathrm{GPa}$ and a yield strength of $730 \mathrm{MPa}$ was successfully fabricated. This combination of high yield strength and low elastic modulus resulted in enhanced elastic recoverable strain of $1.7 \%$, which is much higher than that of the conventional metallic biomaterials. The microstructure responsible for the much sought-after mechanical properties was observed to be mainly consisted of a homogeneous distribution of nanometer-sized $\omega$ - and $\alpha$-precipitates in a $\beta$-phase matrix obtained via cold rolling plus short-time aging at low temperature, i.e. aging at $673 \mathrm{~K}$ for 20 $\mathrm{min}$. These precipitates increase the strength of the material by hindering the motion of dislocations while the $\beta$-matrix with relatively low content of $\beta$-stabilizers gives rise to the observed low elastic modulus. By extending aging time, a higher strength is reached at the expense of an undesirable increasing in elastic modulus.
\end{abstract}

Keywords: $\beta$-type titanium alloy, metallic biomaterials, thermo-mechanical treatments, phase precipitation, elastic deformation behavior.

\section{Introduction}

Titanium and its alloys are normally considered to be the most suitable biomaterials for implant applications due to their excellent biocompatibility and high corrosion resistance ${ }^{1}$. However, the most widely used titanium alloy for implants, $(\alpha+\beta)$-type Ti-6Al-4V, has an elastic modulus $(\sim 110 \mathrm{GPa})$ which is remarkably higher than that of cortical bone tissue ( $\sim 30 \mathrm{GPa})$, leading to the so-called "stress shielding effect" and eventual implant failure ${ }^{2}$. Moreover, $\mathrm{V}$ and $\mathrm{Al}$ ions released from Ti-6Al-4V might induce longterm health problems including Alzheimer's disease ${ }^{3}$. In this scenario, non-toxic metastable $\beta$-type Ti alloys with lower modulus have currently become the hotspots in the field of metallic biomaterials.

The design strategy of $\beta$-type Ti alloys is usually based on the "d-electron alloy design method" proposed by Morinaga ${ }^{4}$. This approach provides a physical background to the phase stability and to the elastic modulus of titanium alloys by connecting the values of two electronic parameters, $B_{o}$ (the covalent bond strength between Ti and alloying elements) and $M_{d}$ (the mean "d" electron-orbital energy level concerning electronegativity and elements radius) to the chemical stability of the phases. Therefore, "alloying vectors" on a $\mathrm{B}_{o}-\mathrm{M}_{d}$ diagram, originating from the $B_{o}$ and $M_{d}$ values of pure Ti, represent the ability of each element to form either stable $\left(\alpha\right.$ and $\beta$ ) or metastable $\left(\alpha^{\prime}, \alpha\right.$ " and $\left.\omega\right)$ phases. Moreover,

*e-mail: athosplaine@hotmail.com since the bonding forces are not only related to the crystal structure, but also to the interatomic distances in the crystal lattice, the elastic modulus of $\beta$-phase is observed to be closely related to its total amount of $\beta$-stabilizers (e.g. $\mathrm{Nb}$, $\mathrm{Ta}, \mathrm{Mo}$, etc.) and decreases monotonically with decreasing total content of $\beta$-stabilizers.

Conversely, insufficient $\beta$-stabilizers might lead to a remarkable decrease in yield strength of these metastable $\beta$-Ti alloys. As such, a precipitation hardening treatment becomes crucial to restore the mechanical strength. However, the second phase precipitates introduced by heat treatments (e.g. annealing) usually exhibit high values of elastic modulus ${ }^{5}$. Additionally, a second phase precipitation might also cause the enrichment of $\beta$-stabilizers in the residual $\beta$-matrix, further increasing the modulus of the $\beta$-phase. As a result, the conventional annealing treatments normally cause an undesirable raise in the elastic modulus of the alloy, making it difficult to simultaneously reach low elastic modulus and high yield strength ${ }^{1}$. Therefore, several studies have been currently carried out with the purpose of finding a method able to strengthen metastable $\beta$-Ti alloys without substantially modifying their elastic modulus ${ }^{6-8}$.

The present study aimed to combine composition design theory and appropriate thermo-mechanical treatments to fabricate a metastable $\beta$-type Ti-Nb-Zr-Ta alloy with concurrently low modulus and high yield strength in order to improve its mechanical compatibility for biomedical applications. Based on the results from X-ray diffraction, transmission electron microscope and tensile test, the relationship between 
microstructural evolution and mechanical properties was discussed.

\section{Materials and Methods}

Homogenized ingots $(140 \mathrm{~mm}$ (length) $\times 25 \mathrm{~mm}$ (width) $\times 20 \mathrm{~mm}$ (thick) ) of Ti-35Nb-7Zr-5 Ta (wt\%) alloy produced by Ercata $\mathrm{GmbH}$ were hot swaged at $1273 \mathrm{~K}$ into rounded bars of $8 \mathrm{~mm}$ in diameter. The chemical composition of the as-received Ti alloy is shown in Table 1. After swaging, samples were solution treated at $1073 \mathrm{~K}$ for $3.6 \mathrm{ks}$ hour under a vacuum of $10^{-5}$ Torr, followed by quenching into water $(\sim 298 \mathrm{~K})$. The specimens that were obtained from the solution treated and water quenched bars were hereafter called as ST specimen. Following quenching, the bars were cold rolled into plates to a final thickness of $\sim 0.9 \mathrm{~mm}$ without intermediate annealing. These plates were then aged at 673 $\mathrm{K}$ for $20 \mathrm{~min}$ and $100 \mathrm{~min}$ and quenched into water $(\sim 298$ $\mathrm{K})$; the corresponding specimens were hereafter called as CRA-20 and CRA-100, respectively.

Phase constitution was determined by X-ray diffractometry (XRD) using a $\mathrm{Cu} \mathrm{K} \alpha$ radiation at an accelerating voltage of $40 \mathrm{kV}$ and a current of $250 \mathrm{~mA}$. Metallographic examinations were initially conducted by conventional optical microscopy (OM). The samples for OM observation were ground, polished, and etched with Kroll solution (distilled water 100 $\mathrm{ml}$, nitric acid $5 \mathrm{ml}$, hydrofluoric acid $3 \mathrm{ml}$ ), consecutively. Some specimens were further characterized by transmission electron microscopy (TEM) at an accelerating voltage of 200 kV (FEI TECNAI G2). TEM samples were mechanically ground to a thickness of approximately $50 \mu \mathrm{m}$ and then ion milled to obtain electron transparency. Selected area electron diffraction (SAD) patterns were indexed using the Java Electron Microscopy Software (JEMS).

Conventional and loading-unloading uniaxial tensile tests were then performed at a cross-head speed of $0.2 \mathrm{~mm} / \mathrm{min}$, using the tensile specimens with 1-mm thickness, 1.46-mm width, and 30-mm gage length. An extensometer was used for accurate strain measurement. Mechanical properties, such as elastic modulus, yield strength and ultimate tensile strength, were determined from the tensile stress-strain curves. In addition to the tensile elastic modulus, the dynamic Young's modulus of the CRA-20 and CRA-100 specimens were also determined by a free resonance vibration technique. Micro-hardness was measured using a Vickers micro-indenter (Zwick Roell ZHV) by loading with a 500 gf load for $15 \mathrm{~s}$ on the samples. Ten different measurements were taken, and the minimum and maximum values were discarded before averaging.

\section{Results and Discussion}

In order to understand the alloying effect on the phase stability of the studied Ti-35Nb-7Zr-5Ta alloy, the electronic parameters $B_{o}$ and $M_{o}$ were first calculated (2.879 and 2.466, respectively) and the alloy was positioned in the $B_{o}-M_{o}$ diagram as shown in Figure 1. Interestingly, the alloy is located in the $\beta+\omega$ region lying below the $\beta / \beta+\omega$ nominal boundary, indicated by a solid line in Figure 1, despite the fact that it is actually a single $\beta$-phase alloy as further confirmed by XRD and TEM analyses. In this particular case, $\mathrm{Zr}$ does not follow the general feature of a neutral element, but instead it behaves as $\beta$-stabilizer when alloyed with other $\beta$-type elements, such as $\mathrm{Nb}$ and $\mathrm{Ta}^{9,10}$. Thus, the coalloying of $\mathrm{Zr}$ shifts the $\beta / \beta+\omega$ phase boundary to the further lower compositional region of $\beta$-stabilizing elements (i.e. $\mathrm{Nb}$ and $\mathrm{Ta}$ ), as indicated by the dashed curve in Figure 1. Furthermore, at this location the Ti-35Nb-7Zr-5Ta alloy has the least stable $\beta$-phase and, consequently, the lowest elastic modulus among $\beta$-phases alloys. The alloy is also close to the transition between several deformation mechanisms predicted for $\beta$-phase. The design procedure followed here considers that large amounts of plastic deformation could be reached as a result of the activation of several mechanically deformation mechanisms (mechanical twinning, stressinduced martensitic transformation and dislocation glide $)^{11}$.

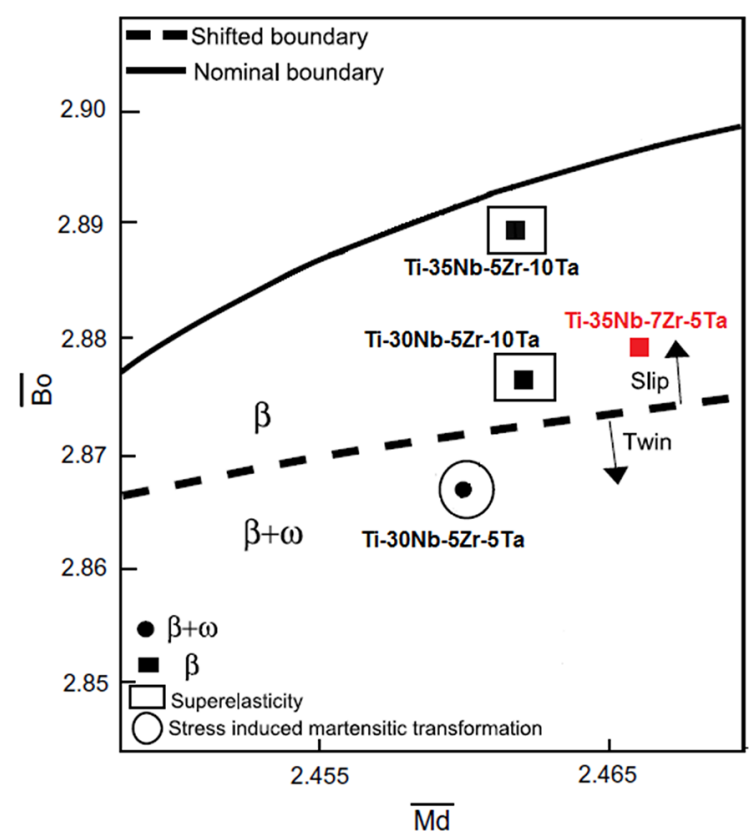

Figure 1. Magnified part of interest of the d-electron alloying map for titanium alloys. The red square indicates the strategic location at which the Ti-35Nb-7Zr-5 Ta (wt\%) Ti alloy is located. (Adapted from Abdel-Hady et al. 2006')

Table 1. Chemical composition of the as-received Ti alloy (wt $\%$ ).

\begin{tabular}{ccccccc}
\hline Ti & Nb & Zr & Ta & O & N & H \\
\hline Bal. & 35.25 & 6.83 & 5.10 & $<0.04$ & $<0.002$ & $<0.001$ \\
\hline
\end{tabular}


Once confirmed, it might give significant support to achieve high levels of cold working and to produce the cold-rolled specimens, from which a large span of mechanical properties can be modulated by heat treatments, based on the diverse phase transformations between $\beta$ and $\alpha$-phases.

Figure 2 shows the XRD patterns of solution treated (ST), CRA-20 and CRA-100 Ti-35Nb-7Zr-5Ta specimens. Only $\beta$ parent phase was observed for the ST condition (Figure 2a), indicating that the alloy exhibits a martensitic start transformation (Ms) temperature lower than room temperature. This can be ascribed to the amount of $\beta$-stabilizers in the present alloy and corroborates to the fact that $\mathrm{Zr}$ behaves as $\beta$-stabilizer in this specific case. For the CRA-20 specimen, Figure $2 b$, a possible second phase formation was not evident by XRD. However, a few $\alpha$ and $\omega$-precipitates start to be detectable in the CRA-100 specimen (Figure 2c), as evidenced by relative weak diffraction peaks of $\alpha(100), \alpha(110), \omega(001), \omega(002)$ and $\omega(112)$. It is known that $\omega$ is a metastable phase that can be formed in Ti- and $\mathrm{Zr}$-based alloys. The athermal $\omega$ phase precipitates after rapid quenching from single $\beta$-phase with body centered cubic (bcc) structure. On the other hand, isothermal $\omega$ structure develops after subsequent annealing at a temperature below $\omega$ solvus. After aging, isothermal $\omega$ is known to promote $\alpha$ phase either directly by acting as heterogeneous nucleation sites or indirectly ${ }^{12,13}$.

The OM and bright-field TEM images of ST specimen are shown in Figure $3 \mathrm{a}$ and $3 \mathrm{~b}$, respectively, confirming that no other phase is formed besides the coarse $\beta$ parent phase. Figure $3 \mathrm{c}$ presents the bright-field TEM image for the CRA-20 specimen, which exhibits a fine microstructure with numerous dark areas caused by a high density of dislocations and grain boundaries. A detail analysis of the SAD pattern in Figure $3 \mathrm{c}$ suggests that besides the primary diffractions from $\beta$, faint additional reflections, attributed to $\alpha$ and $\omega$ phases were also observed. Probably due to low volume fraction of these phases in the CRA-20 condition, $\alpha$ and $\omega$ peaks could not be detected in the XRD pattern shown previously. In order to uncover the morphologies and sizes of these phases,

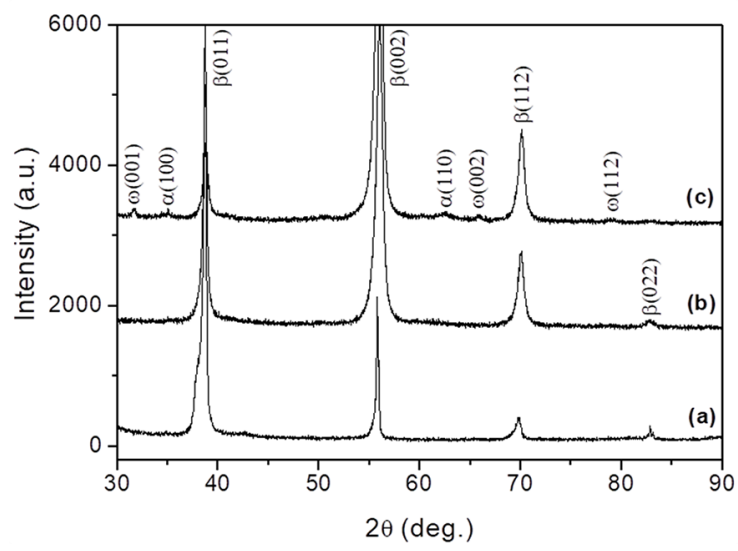

Figure 2. XRD patterns of solution treated (ST) (a), cold-rolled plus 20 min (CRA-20) (b) and $100 \mathrm{~min}$ (CRA-100) (c) aged Ti$35 \mathrm{Nb}-7 \mathrm{Zr}-5 \mathrm{Ta}$ specimens dark-field TEM observation was carried out as presented in Figure $3 \mathrm{~d}$. The $\omega$-precipitates exhibited a more spherical morphology, while the $\alpha$-precipitates exhibited a lenticular morphology, both being nanosized and homogeneously distributed within the $\beta$. The high density of dislocations is assumed to act as heterogeneous nucleation sites, thereby facilitating the unexpected nucleation of isothermal omega at low temperature and short time aging, which can promote alpha nucleation by a displacive mechanism in the core of isothermal omega precipitates ${ }^{14}$. As these heterogeneous nucleation sites are homogenously distributed within the beta matrix, the resulting nanometer-sized precipitates are also homogenously distributed in the entire beta matrix and thus might contribute to an increase in strength of metastable $\beta$ alloys. It is also important to note that the usual $\alpha$ "-martensite of water quenched metastable titanium alloys phase is not detected either by XRD or SAD, which accords with the mutually exclusive formation between the $\omega$ phase and $\alpha^{\prime \prime}$ martensite ${ }^{15}$.

On the other hand, the amount of $\beta$-stabilizers in $\alpha$ precipitates is known to be lower than that in $\beta$ phase of the same Ti alloy ${ }^{16}$. As a result, the precipitation of $\alpha$ within $\beta$-phase, especially for high-temperature and/or long-time aging, usually leads to an enrichment of $\beta$-stabilizers in residual $\beta$-matrix. As previously discussed, this outcome could have created an undesirable effect of increasing the elastic modulus of $\beta$ phase. However, as it will be shown in Figure 4, this was not the case for the CRA-20 specimens.

Figure $4 \mathrm{a}$ gives the representative tensile stress-strain curves of ST and CRA-20 specimens. Apparently, although the ST condition does exhibit low Young's modulus, it is not an effective candidate for structural biomedical applications because of its relatively low yield (180 MPa) and ultimate tensile strength (348 MPa). However, upon cold rolling plus aging treatment, the Ti-35Nb-7Zr-5Ta alloy was remarkably strengthened, with yield strength of $730 \pm 19 \mathrm{MPa}$ and an ultimate tensile strength of $762 \pm 22 \mathrm{MPa}$. By comparison with the conventional metallic implant materials, including 316L SS, tantalum, CP-Ti, Ti6A14V ELI, Nitinol, TiOsteum (a commercial Ti-35Nb-7Zr-5Ta alloy), and $\mathrm{Co}-\mathrm{Cr}$, it can be found that the tensile yield strength of the studied alloy in this condition was close to the tensile yield strength of Ti6Al4V ELI ( 790 MPa), which has the maximum value among these materials ${ }^{6}{ }^{16}$. This strengthening effect is mainly attributed to the interaction between nanometer-sized $\alpha$ and $\omega$ precipitates and high-density dislocations and grain boundaries observed in the microstructure of the CRA-20 specimen. On top of this notable strength level, it should be also observed that the CRA-20 specimen exhibits an ultralow value of Young's modulus of $\sim 47 \mathrm{GPa}$, suggesting that the small amount of the observed nano-precipitates did not cause significant compositional change in $\beta$ matrix. This may be attributed to the present low aging temperature and short aging time, which limits the diffusion of the alloying 

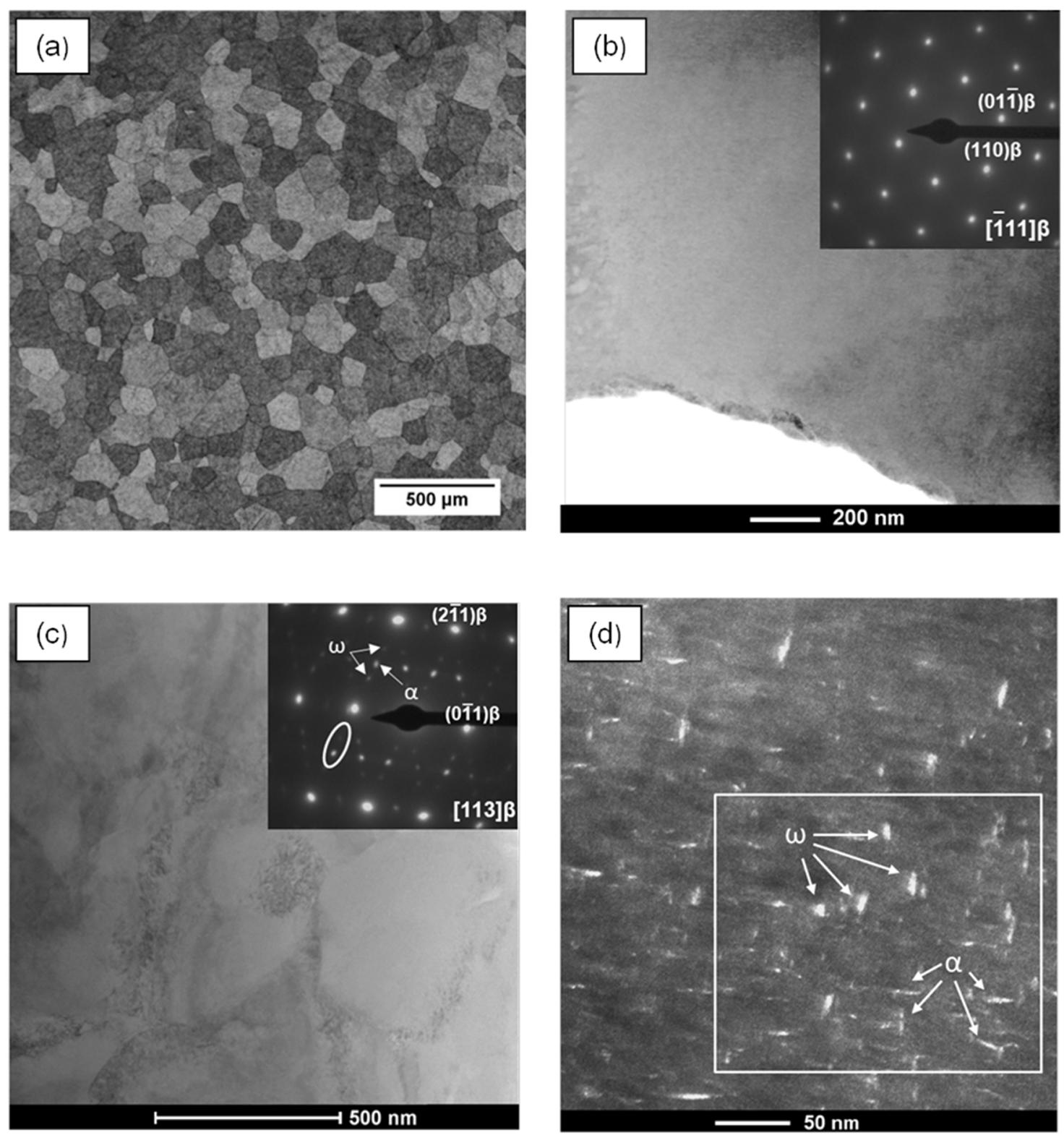

Figure 3. Micrographs of the Ti-35Nb-7Zr-5Ta specimens: (a) OM and (b) TEM bright-field images (and the corresponding SAD pattern) of the solution treated (ST) specimen; (c) TEM bright-field image (and the corresponding SAD pattern) and (d) dark-field image of $\alpha$ and $\omega$ phases of the cold rolled plus 20 min aged (CRA-20) specimen.

elements and, consequently, the size and distribution of the precipitates. To the best of our knowledge this level of modulus is much lower than those of the currently available biomedical Ti alloys ${ }^{1,6}$.

Furthermore, it is also worth to point out that the elastic recoverable strain of the alloy is a valuable parameter in the design of biomedical devices, including self-expansible stents. The higher this parameter, the more desirable the material is for such applications. From the cyclic tensile stress-strain curves of the CRA-20 specimens, presented in Figure 4b, it is observed that in the first cycle (to strain of $1.3 \%$ ), loading and unloading curves are overlapped. The second cycle (to strain of $1.8 \%$ ) is almost completely recovered in spite of a very small residual strain. Lastly, for the third cycle the maximum recoverable strain keeps $1.7 \%$ during unloading at the strain of $2.2 \%$, which is far greater than that of conventional metallic biomaterials such as Ti-6Al$4 \mathrm{~V}(0.79 \%), 316 \mathrm{~L} \mathrm{SS}(0.17 \%)$ and Nitinol $(0.23-0.83 \%)^{6}$.

Figure $4 \mathrm{c}$ shows the elastic modulus and Vickers hardness as a function of different aging times at $673 \mathrm{~K}$. Comparing the $0 \mathrm{~min}$ (i.e., ST specimen) and $20 \mathrm{~min}$ aging conditions, it is possible to note a significant strengthening 


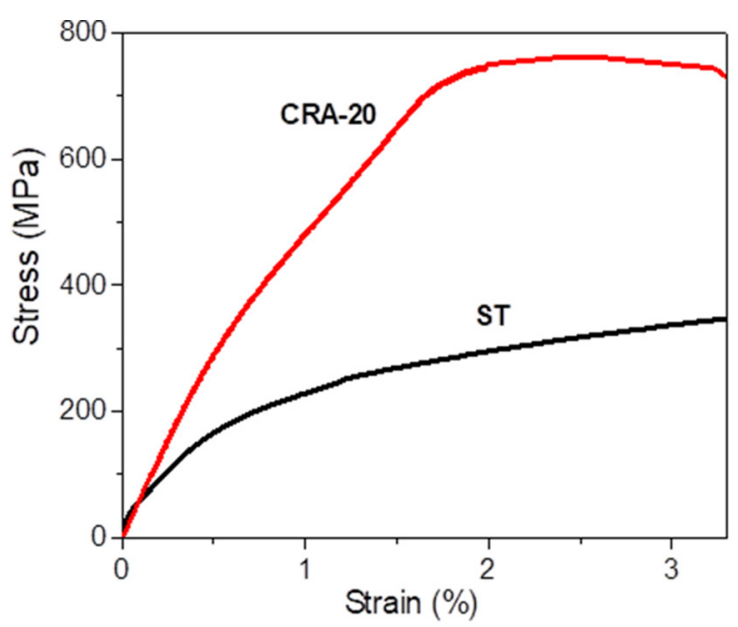

(a)

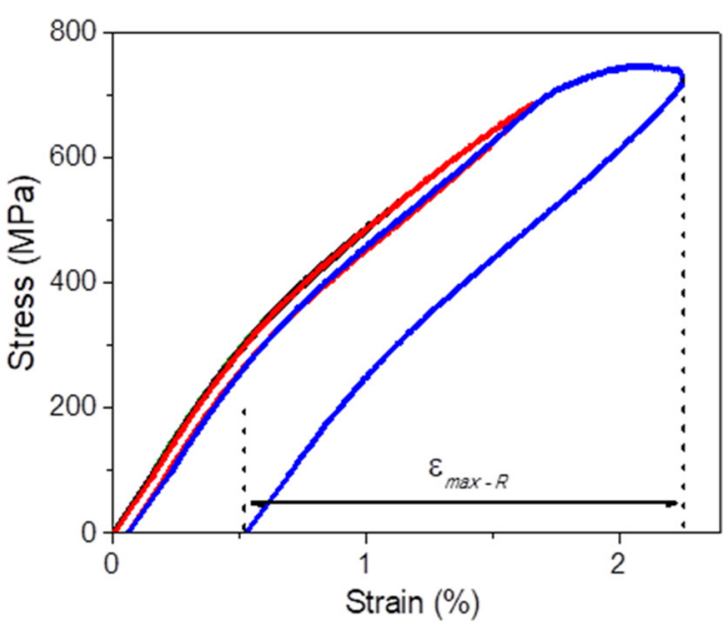

(b)

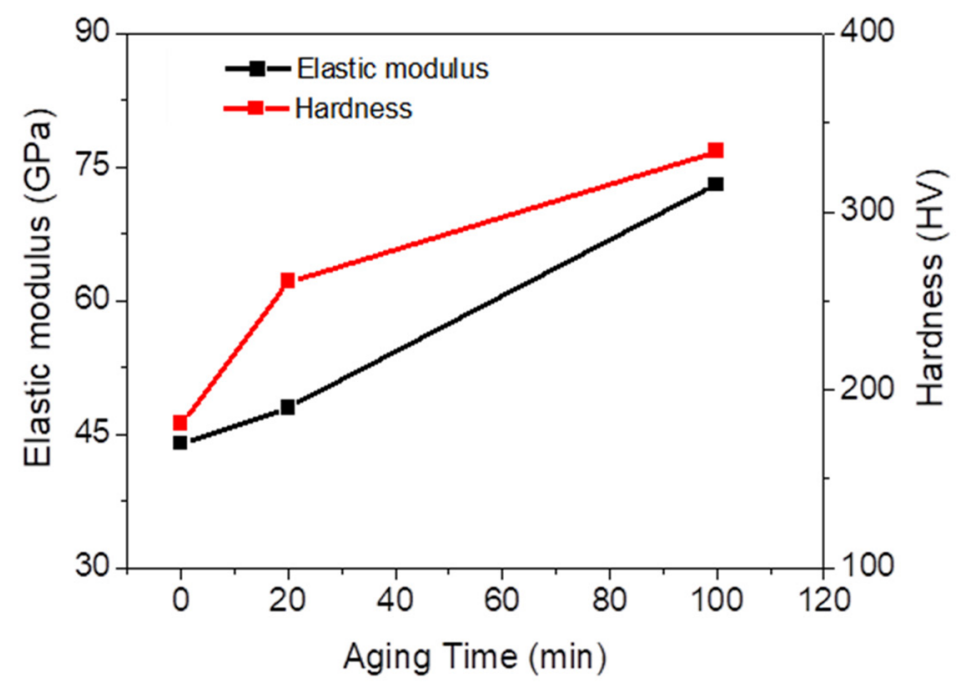

(c)

Figure 4. Mechanical behavior of the Ti-35Nb-7Zr-5Ta specimens: (a) representative tensile stress-strain curves of solution treated (ST) and cold rolled plus 20 min aged (CRA-20) specimens; (b) Cyclic tensile stress-strain curves of the CRA-20 specimen; (c) Elastic modulus and Vickers hardness as a function of different aging times at $673 \mathrm{~K}$.

of the alloy accompanied by a minor increase in elastic modulus, corroborating to the high recoverable strain value previously observed for the CRA-20 specimen. In the case of the 100 min aging condition (CRA-100), a high strength can be obtained at the expense of a similar increase of elastic modulus. Based on these observations, short-time aging at low aging temperature, i.e. aging at $673 \mathrm{~K}$ for $20 \mathrm{~min}$, was confirmed to be the proper aging condition for ensuring ultralow elastic modulus and high yield strength as it limits the amount and size of the second phase precipitates.

\section{Conclusions}

In summary, a metastable $\beta$-type Ti-35Nb-7Zr-5Ta alloy with ultralow elastic modulus and high strength was fabricated by combining composition design theory and an appropriate thermo-mechanical treatment. Although the alloy exhibits low yielding stress in its solution state due to the presence of coarse $\beta$ parent phase, cold rolling plus low temperature/short time aging treatment can result in the combination of relatively high yield stress of $730 \mathrm{MPa}$ and low elastic modulus of $47 \mathrm{GPa}$. The microstructure responsible for the much sought-after mechanical properties consists of a homogeneous distribution of nanometer-sized $\omega$ and $\alpha$ precipitates in a $\beta$-phase matrix. The strengthening effect is attributed to the interaction between the precipitates and the observed high-density dislocations induced by the proposed thermo-mechanical treatments. Meanwhile, a low elastic modulus value is also achieved when only a limited amount of such precipitates is formed in the alloy, to the 
extent that they do not cause significant compositional change in $\beta$-matrix. These findings might have far-reaching consequences by expanding the application range of the biomedical Ti-35Nb-7Zr-5Ta alloy (e.g. structural medical devices such as self-expandable metallic stents) as it exhibits higher strength-to-modulus ratio than those materials currently used in this field.

\section{Acknowledgments}

This work was supported by the São Paulo Research Foundation - FAPESP [grant number 2016/17502-7]; and Coordination for the Improvement of Higher Education Personnel - CAPES.

\section{References}

1. Saini M, Singh Y, Arora P, Arora V, Jain K. Implant biomaterials: A comprehensive review. World Journal of Clinical Cases. 2015;3(1):52-57.

2. Niinomi M, Liu Y, Nakai M, Liu H, Li H. Biomedical titanium alloys with Young's moduli close to that of cortical bone. Regenerative Biomaterials. 2016;3(3):173-185.

3. Gomes CC, Moreira LM, Santos VJSV, Ramos AS, Lyon JP, Soares CP, et al. Assessment of the genetic risks of a metallic alloy used in medical implants. Genetics and Molecular Biology. 2011;34(1):116-121.

4. Morinaga M, Kato M, Kamimura T, Fukumoto M, Harada I, Kubo K. Theoretical Design of b-Type Titanium Alloys. In: Proceedings of Seventh World Conference on Titanium; 1992 Jun 28 - Jul 2; San Diego, CA, USA. Titanium' 92, Science and Technology; 1993. p. 217-224.

5. Banerjee D, Williams JC. Perspectives on Titanium Science and Technology. Acta Materialia. 2013;61(3):844-879.

6. Lin J, Ozan S, Li Y, Ping D, Tong X, Li G, et al. Novel Ti-Ta-Hf$\mathrm{Zr}$ alloys with promising mechanical properties for prospective stent applications. Scientific Reports. 2016;6:37901.
7. Meng Q, Zhang J, Huo Y, Sui Y, Zhang J, Guo S, et al. Design of low modulus ß-type titanium alloys by tuning shear modulus C44. Journal of Alloys and Compounds. 2018;745:579-585.

8. Okulov IV, Volegov AS, Attar H, Bönisch M, Ehtemam-Haghighi $\mathrm{S}$, Calin M, et al. Composition optimization of low modulus and high-strength TiNb-based alloys for biomedical applications. Journal of the Mechanical Behavior of Biomedical Materials. 2017;65:866-871.

9. Abdel-Hady M, Hinoshita K, Morinaga M. General approach to phase stability and elastic properties of ß-type Ti-alloys using electronic parameters. Scripta Materialia. 2006;55(5):477-480.

10. Correa DRN, Kuroda PAB, Lourenço ML, Fernandes CJC, Buzalaf MAR, Zambuzzi WF, et al. Development of Ti-15ZrMo alloys for applying as implantable biomedical devices. Journal of Alloys and Compounds. 2018;749:163-171.

11. Marteleur M, Sun F, Gloriant T, Vermaut P, Jacques PJ, Prima F. On the design of new B-metastable titanium alloys with improved work hardening rate thanks to simultaneous TRIP and TWIP effects. Scripta Materialia. 2012;66(10):749-752.

12. Nag S, Zheng Y, Williams REA, Devaraj A, Boyne A, Wang $\mathrm{Y}$, et al. Non-classical homogeneous precipitation mediated by compositional fluctuations in titanium alloys. Acta Materialia. 2012;60(18):6247-6256.

13. Behera AK. A Study of Mechanisms to Engineer Fine Scale Alpha Phase Precipitation in Beta Titanium Alloy, Beta 21S. [Thesis]. Denton: University of North Texas; 2013.

14. Gloriant T, Texier G, Sun F, Thibon I, Prima F, Soubeyroux JL. Characterization of nanophase precipitation in a metastable $\beta$ titanium-based alloy by electrical resistivity, dilatometry and neutron diffraction. Scripta Materialia. 2008;58(4):271-274.

15. Tian Y, Yu Z, Ong CYA, Kent D, Wang G. Microstructure, elastic deformation behavior and mechanical properties of biomedical B-type titanium alloy thin-tube used for stents. Journal of the Mechanical Behavior of Biomedical Materials. 2015;45:132-141.

16. Boyer R, Collings EW, Welsch G, eds. Materials Properties Handbook: Titanium Alloys. Materials Park: ASM International; 1994. 